\title{
SMALL MAXIMAL SPACES OF NON-INVERTIBLE MATRICES
}

\author{
JAN DRAISMA
}

\section{Abstract}

The (generic) rank of a vector space $\mathcal{A}$ of $n \times n$-matrices is by definition the maximal rank of an element of $\mathcal{A}$. The space $\mathcal{A}$ is called rank-critical if any matrix space that properly contains $\mathcal{A}$ has a strictly higher rank. I present a sufficient condition for rank-criticality, and apply this condition to prove that the images of certain Lie algebra representations are rank-critical.

A rather counter-intuitive consequence is that for infinitely many $n$, there exists an 8-dimensional rank-critical space of $n \times n$-matrices having generic rank $n-1$, or, in other words: an 8-dimensional maximal space of non-invertible matrices. This settles the question, posed by Fillmore, Laurie, and Radjavi in 1985, of whether such a maximal space can have dimension smaller than $n$. As another corollary, I prove that the image of the adjoint representation of a semisimple Lie algebra is rank-critical.

\section{REsults}

This paper deals with linear subspaces of $\operatorname{End}(V)$, the space of $K$-linear maps from an $n$-dimensional vector space $V$ over a field $K$ into itself. The (generic) rank of such a subspace $\mathcal{A}$, denoted rk $\mathcal{A}$, is by definition the highest rank of an element of $\mathcal{A}$, and we call $\mathcal{A}$ rank-critical if any linear subspace $\mathcal{B}$ of $\operatorname{End}(V)$ that properly contains $\mathcal{A}$ has $\operatorname{rk} \mathcal{B}>\operatorname{rk} \mathcal{A}$. Note that a space $\mathcal{A}$ is maximal among the singular spaces - that is, those that only contain non-invertible matrices - if and only if $\mathcal{A}$ is rank-critical of rank $n-1$; in this case we call $\mathcal{A}$ a maximal singular space. The main results of this paper are the following.

Theorem 1. Let $K$ be a field of characteristic zero, let $m$ be an integer $\geq 3$, and let $e$ be a positive integer. Then the image of the representation of $\mathfrak{s l}_{m}(K)$ on the space $V=K\left[x_{1}, \ldots, x_{m}\right]_{e m}$ of homogeneous polynomials of degree em is a maximal singular subspace of $\operatorname{End}(V)$.

In particular, taking $m=3$, we find that for every $n$ of the form $\left(\begin{array}{c}3 e+2 \\ 2\end{array}\right), e>1$ there exists an 8-dimensional maximal singular space of $n \times n$-matrices.

Theorem 2. For any semisimple Lie algebra $\mathfrak{g}$ over a field of characteristic zero, ad $\mathfrak{g}$ is a rank-critical subspace of $\operatorname{End}(\mathfrak{g})$.

Theorems 1 and 2 are consequences of the following proposition.

Date: 14 July 2004.

The author is supported by the Swiss National Science Foundation. 
Proposition 3. Let $\mathcal{A}$ be a subspace of $\operatorname{End}(V)$ and suppose that $|K|>\operatorname{rk} \mathcal{A}=: r$. Set $\mathcal{A}_{\text {reg }}:=\{A \in \mathcal{A} \mid \mathrm{rk} A=r\}$ and define the space

$$
\operatorname{RND}(\mathcal{A}):=\left\{B \in \operatorname{End}(V) \mid B \operatorname{ker} A \subseteq \operatorname{im} A \text { for all } A \in \mathcal{A}_{\text {reg }}\right\} .
$$

Then $\operatorname{RND}(\mathcal{A}) \supseteq \mathcal{A}$, and if equality holds, then $\mathcal{A}$ is rank-critical. If, moreover, a group $G$ acts linearly on $V$ and if $\mathcal{A}$ is stable under the conjugation action of $G$ on $\operatorname{End}(V)$, then $\operatorname{RND}(\mathcal{A})$ is also a $G$-stable.

The proof of Proposition 3 in Section [3 bases on an elementary, but useful sufficient condition for maximality of vector spaces in an arbitrary affine variety embedded in a vector space. In Section 4 we apply Proposition 3 to images of Lie algebra representations; Theorems 1 and 2 are proved there. Section 5 lists some computer results on rank-criticality of semisimple Lie algebra representations - in particular, Theorem 2 came up as a conjecture using this computer program.

Acknowledgments. I thank Matthias Bürgin, Arjeh Cohen, Hanspeter Kraft, Jochen Kuttler, Martijn Stam, and Nolan Wallach for their help and for their interest in the matter of this paper.

\section{InTRODUCTION AND Motivation}

The direct motivation for this paper is the question, posed by Fillmore et al in 1985 [6], of whether a maximal singular subspace of $\operatorname{End}(V)$ can have dimension smaller than $n$. I briefly review three well-known constructions of maximal singular spaces that led them to raise this question.

Example 4. Fix subspaces $W, U$ of $V$ of dimensions $k-1$ and $k$, respectively, and set $\mathcal{A}:=\{A \in \operatorname{End}(V) \mid A U \subseteq W\}$. Then $\mathcal{A}$ is a singular space of dimension $k(k-1)+(n-k) n=n^{2}-k n+k^{2}-k$. Moreover, it not hard to see that $\mathcal{A}$ is maximal. We follow Eisenbud and Harris [5] in calling $\mathcal{A}$ and all its subspaces compression spaces, as they 'compress' $U$ into $W$.

Example 5. Suppose that $n$ is odd, take $V=K^{n}$, and let $\mathcal{A}$ be the space of all skew-symmetric matrices. As any skew-symmetric matrix has even rank, the space $\mathcal{A}$ is singular, and it was observed in [6] that $\mathcal{A}$ is maximal for all odd $n \geq 3$, under the assumption that $|K| \geq 3$. It is easy to see that $\mathcal{A}$ is not a compression space.

In both examples above, the dimension of $\mathcal{A}$ is quadratic in $n$. An ingenious construction of smaller maximal singular spaces is the following, attributed to Bob Paré in [6] and also appearing in [15].

Example 6. Take $V=K^{n}$ and fix $n$ skew-symmetric $n \times n$-matrices $A_{1}, \ldots, A_{n}$. Let $\phi$ be the linear map from $K^{n}$ into the space $M_{n}(K)$ of $n \times n$-matrices over $K$ sending $x$ to the matrix with columns $A_{1} x, A_{2} x, \ldots, A_{n} x$. Then $\phi\left(K^{n}\right)$ is a singular space in $M_{n}(K)$ because $x^{t} \phi(x)=0$ for all $x \in K^{n}$. Moreover, in the particular case where $|K| \geq 3, A_{i}=E_{i, i+1}-E_{i+1, i}$ for $i<n$, and $A_{n}=E_{n, 1}-E_{1, n}$, Fillmore et al showed that $\mathcal{A}$ is maximal $[\underline{6}$.

Many results in the literature exhibit sufficient conditions for a singular space $\mathcal{A}$ to be a compression space: Dieudonné [3] showed that every singular space of dimension $\geq n^{2}-n$ either has a non-trivial common kernel or is dual to a space with a common kernel. Under the assumption that $|K|$ is at least $2 n-2$, this result is sharpened as follows in [6]: if the dimension of $\mathcal{A}$ is $>n^{2}-2 n+2$ (which 
is the dimension of a compression space with $k=2$ ), then $\mathcal{A}$ or its dual has a common kernel. A condition of a different kind is that $\mathcal{A}$ be spanned by rank one matrices; then a combinatorial argument shows that is a compression space 15, 10. Analogues of these questions for (skew-)symmetric matrices and for rank-critical spaces have also been studied extensively in the literature [1, 7, 10, 12, 13, 14, 16].

Yet another result of this type is part of the Kronecker-Weierstrass theory of matrix pencils 9 for $K=\mathbb{C}$. I give a short proof that is valid for other fields, as well.

Proposition 7. Suppose that $|K| \geq n=\operatorname{dim} V$. Then any two-dimensional singular subspace of $\operatorname{End}(V)$ is a compression space.

Proof. If $A, B \in \operatorname{End}(V)$ are such that $K A+K B$ is singular, then the homogeneous polynomial $p(s, t):=\operatorname{det}(s A+t B)$ vanishes on all $|K|+1$ points of $\mathbb{P}^{1}(K)$. As $p(s, t)$ has degree $n<|K|+1$, it must be identically zero, so that $s A+t B$ has a non-zero vector $u(s, t)$ in $K[s, t] \otimes_{K} V$ in its kernel. But then any non-zero homogeneous component of $u(s, t)$, say of degree $d$, is also annihilated by $s A+t B$; hence we find $u_{0}, \ldots, u_{d} \in V$ such that $(s A+t B)\left(s^{d} u_{0}+s^{d-1} t u_{1}+\ldots+t^{d} u_{d}\right)=0$, where we may assume that $u_{0} \neq 0$. Taking the of coefficients of $s^{d+1}, s^{d} t, \ldots, t^{d+1}$, we find

$$
A u_{0}=0, A u_{1}=-B u_{0}, \ldots, A u_{d}=-B u_{d-1} \text {, and } B u_{d}=0 .
$$

But then every element of $K A+K B$ maps the space $U:=\sum_{i} K u_{i}$ into the space $W:=\sum_{i} K A u_{i}$, which is strictly smaller because $A u_{0}=0$ while $u_{0} \neq 0$.

Example 13 below shows that the condition on $|K|$ in this proposition is necessary. This proposition, another proof of which is given in 5 for algebraically closed fields, shows that if $|K| \geq n>2$, then a maximal singular space cannot have dimension 2. On the other hand, Example 6] shows that there do exist maximal singular spaces in $\operatorname{End}(V)$ of dimension $\operatorname{dim}(V)$, and this led Fillmore et al to put forward their question above.

\section{MaXimality of VeCtor SPaCes in AFFine varieties}

Let $K$ be a field and let $M$ be a vector space over $K$. Denote by $L$ an algebraic closure of $K$ and set $M(L):=L \otimes_{K} M$. Let $Z$ be an affine algebraic variety in $M(L)$ defined over $K$, and let $N$ be a $K$-vector subspace of $M$ contained in $Z(K)$. We want a sufficient condition for $N$ to be maximal among the subspaces of $M$ contained in $Z(K)$. Therefore, set

$$
U:=\{m \in M(L) \mid N(L)+L m \subseteq Z\} ;
$$

then $U$ is an affine variety defined over $K$. We make the following assumption:

$$
\forall m \in M(L): N+K m \subseteq Z(K) \Rightarrow m \in U(K) ;
$$

in particular, 0 lies in $U(K)$ and so $N(L) \subseteq Z$.

Lemma 8. Suppose that $Z$ is defined by polynomials $f_{1}, \ldots, f_{k} \in K[M]$. Then $|K|>\max _{i} \operatorname{deg} f_{i}$ implies (*). If, moreover, the $f_{i}$ of highest degree are homogeneous, then $|K| \geq \max _{i} \operatorname{deg} f_{i}$ already implies 因.

Proof. Suppose that $m \in M$ satisfies $N+K m \subseteq Z(K)$, and let $n_{1}, \ldots, n_{d}$ be a $K$-basis of $N$. Then we have for all $i$

$$
f_{i}\left(t_{1} n_{1}+\ldots+t_{d} n_{d}+s m\right)=0 \text { for all } t_{1}, \ldots, t_{d}, s \in K .
$$


But a polynomial of degree $<|K|$ cannot vanish everywhere on an affine space over $K$ unless it is the zero polynomial. Hence if $\operatorname{deg} f_{i}<|K|$, then the left-hand side is the zero polynomial in $t_{1}, \ldots, t_{d}, s$ and vanishes on $L^{d+1}$, as well. We conclude that then $m \in U \cap M=U(K)$, whence the first statement. For the second statement, note that a homogeneous polynomial of degree $\leq K$ cannot vanish everywhere on a projective space over $K$ unless it is the zero polynomial.

By $\circledast$, maximality of $N$ among the subspaces of $M$ contained in $Z(K)$ is equivalent to $U(K)=N$, and a sufficient condition for this is clearly $U=N(L)$. In principle, this condition can be verified using Gröbner basis techniques, but $U$ may be hard to compute even for moderately complicated $Z$. We therefore set out to find linear sufficient conditions, as follows: Let $Z_{\text {reg }}$ be the set of smooth points of $Z$, and set $N_{\text {reg }}:=N \cap Z_{\text {reg }}, N(L)_{\text {reg }}:=N(L) \cap Z_{\text {reg }}$. The second assumption we make is

$$
N_{\text {reg }} \neq \emptyset
$$

Now let

$$
T_{N(L)_{\mathrm{reg}}} Z:=\bigcap_{n \in N(L)_{\mathrm{reg}}} T_{n} Z
$$

be the intersection of all tangent spaces to $Z$ at points of $N(L)_{\text {reg }}$, where each $T_{n} Z$ is viewed as a vector subspace (through the origin) of $M(L)$. Then we have the following lemma.

Lemma 9. The L-vector space $T_{N(L)_{r e g}} Z$ contains $U$. In particular, if $T_{N(L)_{r e g}} Z=$ $N(L)$, then $N(L)$ is maximal among the $L$-subspaces of $M(L)$ contained in $Z$.

Proof. For $m \in U$ and $n \in N(L)_{\text {reg }}$ the line $\{n+t m \mid t \in L\}$ lies in $Z$, and $a$ fortiori $m$ is tangent to $Z$ at $n$. We conclude that $m \in T_{N(L)_{\mathrm{reg}}} Z$, as claimed. The second statement is immediate.

We want a $K$-rational version of this lemma. To this end, we define

$$
T_{N_{\mathrm{reg}}}(Z(K)):=\bigcap_{n \in N_{\mathrm{reg}}}\left(\left(T_{n} Z\right)(K)\right)=\left(\bigcap_{n \in N_{\mathrm{reg}}} T_{n} Z\right)(K),
$$

where the second equality follows from the fact that $Z$, and therefore every $T_{n} Z$, is defined over $K$. Note that $T_{N_{\mathrm{reg}}}(Z(K))$ contains $\left(T_{N(L)_{\mathrm{reg}}} Z\right)(K)$, as the intersection is taken over a smaller set.

Proposition 10. The $K$-vector space $T_{N_{\text {reg }}}(Z(K))$ contains $U(K)$. In particular, if $T_{N_{\text {reg }}}(Z(K))=N$, then $N$ is maximal among the $K$-vector subspaces of $M$ contained in $Z(K)$.

Proof. If $m \in U(K)$, then by the previous lemma $m \in\left(T_{N(L)_{\mathrm{reg}}} Z\right)(K)$, which space is contained in $T_{N_{\mathrm{reg}}}(Z(K))$; this shows the first statement. The second statement is now immediate from assumption $\$$.

A randomised algorithm to compute the tangent space $T_{N_{\mathrm{reg}}} Z(K)$ bases on the following observation.

Proposition 11. For all non-negative integers $l$ and $e$, the set of $\left(n_{1}, \ldots, n_{l}\right) \in$ $N_{\text {reg }}^{l}$ for which

$$
\operatorname{dim}_{K} \bigcap_{i=1}^{l}\left(T_{n_{i}} Z\right)(K) \geq e
$$


is the set of $K$-rational points of a closed subvariety of $N(L)_{\text {reg }}^{l}$.

Proof. Let $d$ be the dimension of $Z$ and let $\gamma: Z_{\text {reg }} \rightarrow \operatorname{Gr}_{d}(M(L))$ be the Gauss map sending a point of $Z$ to its tangent space. Now the set of all $l$-tuples $\left(T_{1}, \ldots, T_{l}\right) \in$ $\mathrm{Gr}_{d}(M(L))^{l}$ whose intersection has dimension at least $e$ is closed in $\operatorname{Gr}_{d}(M(L))^{l}$, hence so is its pre-image under $\left(\left.\gamma\right|_{N_{\text {reg }}}\right)^{\times l}$. The set of the proposition is the set of $K$-rational points of this pre-image.

For dimension reasons, the space $T_{N_{\text {reg }}} Z(K)$ is the intersection of finitely many tangent spaces $T_{n_{i}} Z(K), i=1, \ldots, m$ with $n_{i} \in N_{\text {reg. Now }} K$ is large (in particular, if $K$ is infinite), then the preceding proposition suggests the following randomised algorithm to compute $T_{N_{\text {reg }}} Z(K)$ : First, find an upper bound on $m$, and second, choose $m$ elements of $N$ at random. These are probably smooth points by $* *$, and by the preceding proposition the intersection of their tangent spaces is probably equal to $T_{N_{\text {reg }}} Z(K)$. In particular, if this intersection is equal to $N$, then you are sure that $N$ is a maximal vector space in $Z(K)$. This algorithm was used to produce computational evidence for Theorem 2 see Section 5 For the second half of Proposition 3 we need the following.

Proposition 12. Suppose that a group $G$ acts $K$-linearly on $M$, that $N$ is $G$ stable, and that $Z$ is stable under the corresponding $L$-linear action on $M(L)$. Then $T_{N_{\text {reg }}} Z(K)$ is a $G$-submodule of $M$.

Proof. Let $m \in T_{N_{\text {reg }}} Z(K), g \in G$, and $n \in N_{\text {reg. }}$ Then $g^{-1} n \in N \cap Z_{\text {reg }}=$ $N_{\text {reg }}$, and $g$ maps the $K$-rational tangent space $\left(T_{g^{-1} n} Z\right)(K)$ isomorphically onto $\left(T_{n} Z\right)(K)$. As $m$ lies in the former by assumption, $g m$ lies in the latter. This shows that $g m \in T_{N_{\text {reg }}} Z(K)$.

We will apply Proposition 10 to the setting of Section 11 $M=\operatorname{End}(V)$, where $V$ is a vector space over $K, N=\mathcal{A}$ is a subspace of $\operatorname{End}(V)$ of generic rank $r$, and $Z=R_{r}$ is the variety of $L$-linear maps $V(L) \rightarrow V(L)$ of rank at most $r$. The following example shows that assumption (*) is not automatic here.

Example 13. Suppose that $K$ is a finite field with $q$ elements labelled $c_{1}, \ldots, c_{q}$. Then the 2-dimensional subspace $\mathcal{A}$ of $M_{q+1}(K)$ spanned by the diagonal matrices $A=\operatorname{diag}(1,1, \ldots, 1,0)$ and $B=\operatorname{diag}\left(c_{1}, c_{2}, \ldots, c_{q}, 1\right)$ is singular-i.e., lies in $R_{q}(K)$-but $\mathcal{A}(L)$ is not.

We avoid this anomaly as follows: $R_{r}$ is defined by the $(r+1) \times(r+1)$-minors of matrices, which are homogeneous polynomials of degree $r+1$. Hence, if we assume that $|K| \geq r+1$, then assumption (*) is automatically fulfilled by Lemma 8 in particular, the rank of $\mathcal{A}(L)$ is then also $r$. Moreover, the smooth points of $R_{r}$ are the linear maps of rank exactly $r$, and by assumption $\mathcal{A}$ contains such maps, so that assumption (**) is also satisfied. The space $T_{\mathcal{A}_{\mathrm{reg}}} R_{r}(K)$ will be denoted $\operatorname{RND}(\mathcal{A})$, and its elements will be called rank-neutral directions of $\mathcal{A}$. We recall a useful characterisation of the tangent spaces to $R_{r}$ at smooth points.

Lemma 14. For $A \in R_{r}(K)$ of rank $r$, the tangent space $\left(T_{A} R_{r}\right)(K)$ is equal to $\{B \in \operatorname{End}(V) \mid B \operatorname{ker} A \subseteq \operatorname{im} A\}$.

Proof. This is well known [11, Example 14.16]; I only give an intuitive argument: For $B$ to lie in $T_{A} R_{r}$, it is necessary and sufficient that there be, for every $v \in \operatorname{ker}(A)$, a vector $w \in V$ for which $(A+\epsilon B)(v+\epsilon w)=0$ modulo $\epsilon^{2}$. The coefficient of $\epsilon$ 
in this expression is $A w+B v$, so that the existence of such a $w$ is equivalent to $B v \in \operatorname{im}(A)$.

Proposition 3 is now a direct consequence of Proposition [10 Lemma 14 and Proposition 12

Example 15. Proposition 3 provides another proof of rank-criticality of compression spaces (under the condition $|K| \geq n$ ). Indeed, in the notation of Example 4 suppose that $B \in \operatorname{End}(V)$ does not map $U$ into $W$, and let $u \in U$ be such that $B u \notin W$. It is not hard to construct an $A \in \mathcal{A}$ with $\operatorname{ker} A=K u$ and $\operatorname{im} A \not \supset B u$. But then $B \notin\left(T_{A} R_{n-1}\right)(K)$ by Lemma 14 and we conclude that $\operatorname{RND}(\mathcal{A})=\mathcal{A}$.

\section{A CONSTRUCTION OF RANK-CRITICAL SPACES}

The singular space of Example 5 is closed under the commutator, and so are the compression spaces of Example 4 if $W \subseteq U{ }^{1}$ This suggests the study of the following situation: Let $G$ be an affine algebraic group defined over $K$, and let $\rho: G \rightarrow \mathrm{GL}_{K}(V)$ be a finite-dimensional representation defined over $K$. Let $\mathfrak{g}$ be the Lie algebra of $G$, and denote the corresponding representation $\mathfrak{g} \rightarrow \operatorname{End}(V)$ also by $\rho$. Set $r:=\operatorname{rk} \rho(\mathfrak{g})$ and suppose that $|K| \geq r+1$. Now $G$ acts on $\operatorname{End}(V)$ by $g A:=\rho(g) A \rho(g)^{-1}$, and both $\rho(\mathfrak{g})$ and the variety $R_{r}$ of linear maps of rank $\leq r$ are $G$-stable. Proposition 3 implies: the rank-neutral directions of $\rho(\mathfrak{g})$ form a $G$-module, and if $\operatorname{RND}(\rho(\mathfrak{g}))=\rho(\mathfrak{g})$, then $\rho(\mathfrak{g})$ is rank-critical. In the rest of this section we assume that char $K=0$, so that we can use the well-known representation theory of semisimple Lie algebras to construct rank-critical spaces.

Example 16. Recall Example 5. Here $G$ is the group $\mathrm{O}_{n}$ of orthogonal matrices, $\mathfrak{g}=\mathfrak{o}_{n}, \rho$ is the identity, and $V=K^{n}$ is the standard $\mathfrak{o}_{n}$-module. It is well known that $\operatorname{End}(V)$ is the direct sum of three irreducible $\mathrm{O}_{n}$-modules: the space $\mathfrak{o}_{n}$ of skew-symmetric matrices, the scalar multiples of the identity $I$, and the space of symmetric matrices with trace 0 . We now use Lemma 14 to show that the last two modules are not contained in $\operatorname{RND}\left(\mathfrak{o}_{n}\right)$ : Choose

$$
Y:=\operatorname{diag}(1,-1,0, \ldots, 0) \text { and } X:=\left[\begin{array}{c|c}
0 & 0 \\
\hline 0 & X^{\prime}
\end{array}\right],
$$

where $X^{\prime} \in \mathfrak{o}_{n-1}$ has full rank $n-1$. Then neither $I$ nor $Y$ maps ker $X$ into $\operatorname{im} X$, hence the $\mathrm{O}_{n}$-modules that they represent are not contained in $\operatorname{RND}\left(\mathfrak{o}_{n}\right)$. We conclude that $\operatorname{RND}\left(\mathfrak{o}_{n}\right)=\mathfrak{o}_{n}$, so that $\mathfrak{o}_{n}$ is maximal singular.

Suppose now that $\mathfrak{g}$ is semisimple and that it has a split Cartan subalgebra $\mathfrak{h}$. Then $V$ is the direct sum of its $\mathfrak{h}$-weight spaces $V_{\lambda}, \lambda \in \mathfrak{h}^{*}$, and we have $r=$ $\operatorname{dim} V-\operatorname{dim} V_{0}$. Furthermore, we choose a Borel subalgebra $\mathfrak{b}$ of $\mathfrak{g}$ containing $\mathfrak{h}$. We can then compute, for each $\mathfrak{b}$-highest weight $\lambda$ of $\operatorname{End}(V)$, the multiplicity of $\lambda$ among the highest weights in $\operatorname{RND}(\rho(\mathfrak{g}))$; see Section 5

If, moreover, $K$ is algebraically closed, then the rank-neutral directions of $\rho(\mathfrak{g})$ can be characterised as follows:

$$
\operatorname{RND}(\rho(\mathfrak{g}))=\left\{Y \in \operatorname{End}(V) \mid(g Y) V_{0} \subseteq \bigoplus_{\lambda \neq 0} V_{\lambda} \text { for all } g \in G\right\} .
$$

\footnotetext{
${ }^{1}$ Note that this property is not preserved under the multiplication from the left and from the right with arbitrary invertible matrices - however, deciding whether a given subspace $\mathcal{A}$ of $\operatorname{End}(V)$ is $\mathrm{GL}(V) \times \mathrm{GL}(V)$-conjugate to a Lie algebra is easily reduced to a linear problem.
} 
Indeed, a generic element of $\rho(\mathfrak{h})$ has kernel $V_{0}$ and image $V_{1}:=\bigoplus_{\lambda \neq 0} V_{\lambda}$, so that the inclusion $\subseteq$ follows from Lemma 14. On the other hand, if any map in the $G$-orbit of $Y$ maps $V_{0}$ into $V_{1}$, then $Y$ is tangent to $R_{r}$ at all points of $\left\{g X \mid g \in G, X \in \rho(\mathfrak{h})\right.$ with $\operatorname{ker} X=V_{0}$ and $\left.\operatorname{im} X=V_{1}\right\}$. This set is dense in $\rho(\mathfrak{g})_{\text {reg }}$, and because the set $\left\{X \in \rho(\mathfrak{g})_{\text {reg }} \mid Y \in T_{X} R_{r}\right\}$ is closed in $\rho(\mathfrak{g})_{\text {reg }}$, we conclude that $Y \in \operatorname{RND}(\rho(\mathfrak{g}))$. We now proceed with the proof of Theorem 1

Let $\mathfrak{g}$ be the Lie algebra $\mathfrak{s l}_{m}(K)$, let $k$ be a natural number, and let $\rho: \mathfrak{g} \rightarrow$ $\operatorname{End}(V)$ be the representation of $\mathfrak{g}$ on the $k$-th symmetric power $V=S^{k}\left(\left(K^{m}\right)^{*}\right)$ of $\left(K^{m}\right)^{*}$, i.e., on the homogeneous polynomials on $K^{m}$ of degree $k$. Let $\mathfrak{h} \subseteq \mathfrak{g}$ be the Cartan subalgebra of diagonal matrices and let $\mathfrak{b} \supseteq \mathfrak{h}$ be the Borel subalgebra of upper triangular matrices. The image of $\rho$ is spanned by the (restrictions to $V$ of the) differential operators $x_{i} \frac{\partial}{\partial x_{j}}, i \neq j$, and $x_{i} \frac{\partial}{\partial x_{i}}-x_{j} \frac{\partial}{\partial x_{j}}$, where the latter span $\rho(\mathfrak{h})$. The weight spaces in $V$ are one-dimensional and spanned by the monomials $x_{1}^{a_{1}} \cdots x_{m}^{a_{m}}$ with $a_{1}+\ldots+a_{m}=k$. The highest root vector in $\rho(\mathfrak{g})$ is $x_{m} \frac{\partial}{\partial x_{1}}$. To apply Proposition 3 we compute the highest weight vectors in $\operatorname{End}(V)$.

Lemma 17. The highest weight vectors in $\operatorname{End}(V)=V \otimes V^{*}$ are precisely the powers $\left(x_{m} \frac{\partial}{\partial x_{1}}\right)^{d}$ for $d=0, \ldots, k$.

Proof. It is clear that these are (non-zero) highest weight vectors; that there are no others follows by a dimension computation. Alternatively, the lemma is an easy application of the Littlewood-Richardson rule [8].

The space $\rho(\mathfrak{g})$ is singular if and only if $k$ is a multiple of $m$, say $k=e m$, and then $x_{1}^{e} x_{2}^{e} \ldots x_{m}^{e}$ spans the zero weight space. The space $\rho\left(\mathfrak{s l}_{m}\right)$ has no chance of being maximal singular if $m=2$ (unless $\mathrm{k}=2$ ), as then $\operatorname{dim}(V)=k+1=2 e+1$ is odd and $\mathrm{SL}_{2}$ leaves invariant a non-degenerate symmetric bilinear form on $V ; \rho(\mathfrak{g})$ is then contained in the larger singular space of linear maps that are skew relative to this bilinear form. This explains the condition $m \geq 3$ in Theorem 1

Proof of Theorem [1. First, if the image of $\mathfrak{g}$ is maximal singular over a larger field, then it is also maximal singular over $K$; so it suffices to prove the theorem for $K$ algebraically closed. By Proposition 3 Lemma 17, and the characterisation *** of $\operatorname{RND}(\rho(\mathfrak{g}))$ it suffices to prove that if $d \neq 1$, then some element of the $\mathrm{SL}_{m}$-orbit of $\left(x_{m} \frac{\partial}{\partial x_{1}}\right)^{d}$ does not map $x_{1}^{e} \cdots x_{m}^{e}$ into the space spanned by all other monomials. This $\mathrm{SL}_{m}$-orbit contains the differential operators of the form $\left(x_{1}+x_{2}+x_{3}\right)^{d}\left(\alpha \frac{\partial}{\partial x_{1}}+\right.$ $\left.\beta \frac{\partial}{\partial x_{2}}+\gamma \frac{\partial}{\partial x_{3}}\right)^{d}$ with $\alpha+\beta+\gamma=0$, and the coefficient of $x_{1}^{e} \cdots x_{m}^{e}$ in

$$
\left(x_{1}+x_{2}+x_{3}\right)^{d}\left(\alpha \frac{\partial}{\partial x_{1}}+\beta \frac{\partial}{\partial x_{2}}+\gamma \frac{\partial}{\partial x_{3}}\right)^{d} x_{1}^{e} \cdots x_{m}^{e}
$$

is equal to

$$
\sum_{a, b, c: a+b+c=d}\left(\frac{d !}{a ! b ! c !}\right)^{2}(e)_{a}(e)_{b}(e)_{c} \alpha^{a} \beta^{b} \gamma^{c},
$$

where $(e)_{p}:=e(e-1) \cdots(e-p+1)$ is the falling factorial. Lemma 18 below shows that this polynomial in $\alpha, \beta$, and $\gamma$ is not a multiple of $\alpha+\beta+\gamma$ if $d \neq 1$. In particular, for all $d \neq 1$ there exist $\alpha, \beta, \gamma \in K$ with $\alpha+\beta+\gamma=0$ for which the coefficient above is non-zero, that is, for which the term $x_{1}^{e} \cdots x_{m}^{e}$ does indeed occur in $\left(x_{1}+x_{2}+x_{3}\right)^{d}\left(\alpha \frac{\partial}{\partial x_{1}}+\beta \frac{\partial}{\partial x_{2}}+\gamma \frac{\partial}{\partial x_{3}}\right)^{d} x_{1}^{e} \cdots x_{m}^{e}$; this concludes the proof. 
Lemma 18. For any integers $e>0$ and $d \in\{0\} \cup\{2,3, \ldots, 3 e\}$ the polynomial

$$
P_{d, e}(\alpha, \beta, \gamma):=\sum_{a, b, c:} a+b+c=d \quad \frac{d !}{a ! b ! c !}\left(\begin{array}{l}
e \\
a
\end{array}\right)\left(\begin{array}{l}
e \\
b
\end{array}\right)\left(\begin{array}{l}
e \\
c
\end{array}\right) \alpha^{a} \beta^{b} \gamma^{c} \in \mathbb{Z}[\alpha, \beta, \gamma]
$$

is not divisible by $\alpha+\beta+\gamma$.

Proof. First, $P_{0, e}=1$ is not divisible by $\alpha+\beta+\gamma$; suppose next that $d \geq 2 e$. Then the highest monomial in $P_{d, e}$ with respect to the lexicographic order with $\alpha>$ $\beta>\gamma$ is $\alpha^{e} \beta^{e} \gamma^{d-2 e}$. Rewriting $P_{d, e}$ as a polynomial in the elementary symmetric polynomials $\sigma_{1}:=\alpha+\beta+\gamma, \sigma_{2}:=\alpha \beta+\beta \gamma+\gamma \alpha$, and $\sigma_{3}:=\alpha \beta \gamma$ will therefore give the monomial $\sigma_{3}^{d-2 e} \sigma_{2}^{3 e-d}$ a non-zero coefficient. Hence $P$ is not divisible by $\sigma_{1}$.

If $1<d<2 e$, then we claim that the coefficient of $\alpha^{d-1} \beta$ in $P_{d, e}(\alpha, \beta,-\alpha-\beta)$ is non-zero. Indeed, this coefficient is readily seen to equal $Q_{d-1}$, where

$$
Q_{d}:=(e+1)(d+1) \sum_{a=0}^{d}\left(\begin{array}{l}
d \\
a
\end{array}\right)\left(\begin{array}{l}
e \\
a
\end{array}\right)\left(\begin{array}{c}
e \\
d-a
\end{array}\right)(-1)^{a} \frac{a}{a+1}, \quad d \geq 1 .
$$

Automatic summation using Zeilberger-Wilf theory and the Maple-package EKHAD8 2] 17. yields the following expression for $Q_{d}$ :

$$
Q_{d}= \begin{cases}(-1)^{k} \frac{(e+k)(e+k-1) \cdots(e-k+1)}{k \cdot((k-1) !)^{2}} & \text { if } d=2 k-1, \text { and } \\ (-1)^{k}(2 e+1) \frac{(e+k)(e+k-1) \cdots(e-k+1)}{k \cdot((k-1) !)^{2}} & \text { if } d=2 k .\end{cases}
$$

In particular, we find that $Q_{d}$ is non-zero for $1 \leq k \leq e$, so that the coefficient of $\alpha^{d-1} \beta$ in $P_{d, e}(\alpha, \beta,-\alpha-\beta)$ is in fact non-zero for $2 \leq d \leq 2 e+1$. This concludes the proof of the lemma.

We now prove rank-criticality of the images of adjoint representations.

Proof of Theorem [2] Again, it suffices to prove the theorem in the case where $K$ is algebraically closed. We will prove $\operatorname{RND}(\operatorname{ad}(\mathfrak{g}))=\operatorname{ad}(\mathfrak{g})$ and then apply Proposition 3. Therefore, let $A$ be a rank-neutral direction of ad( $\mathfrak{g})$ and let $x \in \mathfrak{g}$ have centraliser $\mathfrak{g}^{x}$ of minimal dimension; then $A \mathfrak{g}^{x} \subseteq[x, \mathfrak{g}]$ by Lemma 14. In particular, we find that if $[x, y]=0$, then the Killing form $\kappa$ of $\mathfrak{g}$ vanishes on $(x, A y)$. As the commuting variety of $\mathfrak{g}$ is irreducible [18, this implies that $\kappa(x, A y)=0$ for all $x, y \in \mathfrak{g}$ with $[x, y]=0$, independent of $\operatorname{dim} \mathfrak{g}^{x}$. Now consider the space

$$
M(\mathfrak{g}):=\{A \in \operatorname{End}(\mathfrak{g}) \mid \kappa(x, A y)=0 \text { for all } x, y \in \mathfrak{g} \text { with }[x, y]=0\} ;
$$

we claim that it is equal to ad $(\mathfrak{g})$. First, assume that this is true for simple $\mathfrak{g}$, let $\mathfrak{g}=\bigoplus_{i} \mathfrak{g}_{i}$ be a decomposition of $\mathfrak{g}$ into simple ideals, let $A \in M(\mathfrak{g})$, and let $y \in \mathfrak{g}_{i}$. Then $A y$ is $\kappa$-perpendicular to $\bigoplus_{j \neq i} \mathfrak{g}_{j}$, so $A y \in \mathfrak{g}_{i}$. In other words, every $\mathfrak{g}_{i}$ is $A$-stable, and of course $\left.A\right|_{\mathfrak{g}_{i}} \in M\left(\mathfrak{g}_{i}\right)$. By assumption there exist $z_{i} \in \mathfrak{g}_{i}$ such that $\left.A\right|_{\mathfrak{g}_{i}}=\operatorname{ad}_{\mathfrak{g}_{i}} z_{i}$, and then $A=\sum_{i} \operatorname{ad}_{\mathfrak{g}}\left(z_{i}\right)$.

It remains to prove $M(\mathfrak{g})=\operatorname{ad}(\mathfrak{g})$ for simple $\mathfrak{g}$. For $\mathfrak{s l}_{2}$ this is easy, so we may suppose that $\mathfrak{g}$ has rank $\geq 2$. Setting $x=y$ in the condition on $A$, we see that $M(\mathfrak{g}) \subseteq \mathfrak{o}(\kappa)$, the orthogonal Lie algebra defined by $\kappa$. Moreover, $M(\mathfrak{g})$ is stable under conjugation with any automorphism of $\mathfrak{g}$, and this implies two things: first, that $M(\mathfrak{g})$ is a $\mathfrak{g}$-module and second, using the Chevalley involution of $\mathfrak{g}$, that it is self-dual as such. Now the $\mathfrak{g}$-module $\mathfrak{o}(\kappa) / \operatorname{ad}(\mathfrak{g})$ is irreducible if $\mathfrak{g}$ is not of type $A$ its highest weight can be determined explicitly but is not of interest to us - while it 
is a direct sum $W \oplus W^{*}$ for some non-self-dual module $W$ if $\mathfrak{g}$ is of type $A$. In any case, $M(\mathfrak{g})$ is either ad $(\mathfrak{g})$ or $\mathfrak{o}(\kappa)$. But $M(\mathfrak{g}) \neq \mathfrak{o}(\kappa)$ : choose for instance $x, y$ in (a Cartan subalgebra of) $\mathfrak{g}$ satisfying $[x, y]=0$ and $\kappa(x, x)=\kappa(y, y)=1-\kappa(x, y)=1$ and let $A$ be the map sending $x$ to $y, y$ to $-x$, and the $\kappa$-orthogonal complement of $\langle x, y\rangle_{K}$ to 0 ; then $A \in \mathfrak{o}(\kappa) \backslash M(\mathfrak{g})$. We conclude that $M(\mathfrak{g})=\operatorname{RND}(\operatorname{ad}(\mathfrak{g}))=\operatorname{ad}(\mathfrak{g})$, as claimed.

\section{Some COMPUter RESUlts}

To compute the $G$-module $\operatorname{RND}(\rho(\mathfrak{g}))$, where $\rho: \mathfrak{g} \rightarrow \operatorname{End}(V)$ is a finitedimensional representation of a split semisimple Lie algebra $\mathfrak{g}$ over a field $K$ of characteristic 0, we use the following algorithm based on Proposition 11

(1) Compute the non-zero highest weight spaces $\mathrm{HW}_{\mu}$ of $\operatorname{End}(V)$ relative to a Borel subalgebra $\mathfrak{b}$ and a split Cartan subalgebra $\mathfrak{h}$ contained in $\mathfrak{b}$; this is elementary linear algebra.

(2) For each of them, say $\mathrm{HW}_{\mu}$ of dimension $l$, choose $l$ random elements $X_{1}, \ldots, X_{l} \in \rho(\mathfrak{g})$, verify that they have maximal rank, and compute the subspace

$$
\left\{Y \in \mathrm{HW}_{\mu} \mid Y \operatorname{ker} X_{i} \subseteq \operatorname{im} X_{i} \text { for all } i=1, \ldots, l\right\} .
$$

(3) The dimension of this space is the multiplicity of $\mu$ among the highest weights in $\operatorname{RND}(\rho(\mathfrak{g}))$. Well, more precisely, it is an upper bound to this multiplicity, which with high probability is sharp.

If we find that the multiplicity of every highest weight in $\operatorname{RND}(\rho(\mathfrak{g}))$ is equal to its multiplicity in $\rho(\mathfrak{g})$, then $\rho(\mathfrak{g})$ is rank-critical by Proposition 3 I list some examples, found by computer experiments with this algorithm.

(1) The images of the adjoint representations of split simple Lie algebras of types $A_{1}, \ldots, A_{4}, B_{2}, \ldots, B_{4}, C_{3}, C_{4}$, and $G_{2}$ were proved rank-critical with this algorithm. This computational evidence led to the formulation of Theorem 2

(2) The image of the 26-dimensional representation of $F_{4}$ is rank-critical (of rank 24).

(3) Let $\mathfrak{g}$ be split simple of type $G_{2}$ and let $\rho$ be the 7-dimensional representation of highest weight $[1,0]$, with zero weight multiplicity 1 . Then $\operatorname{RND}(\rho(\mathfrak{g}))$ is equal to $\mathfrak{o}_{7}$, which of course is still singular, so $\rho(\mathfrak{g})$ is not a maximal singular space.

(4) Similarly, if $\mathfrak{g}$ is of type $G_{2}$ and $\rho$ is the 27-dimensional representation of highest weight $[2,0]$ with zero weight multiplicity 3 , then $\operatorname{RND}(\rho(\mathfrak{g}))$ is equal to $\phi\left(\mathfrak{o}_{7}\right)$, where $\phi$ is the representation of $\mathfrak{o}_{7}$ of highest weight $[2,0,0]$, which restricts to $\rho$ on $\mathfrak{g}$. As both $\rho(\mathfrak{g})$ and $\phi\left(\mathfrak{o}_{7}\right)$ have generic rank 24 , the former is not rank-critical but the latter is.

(5) Let $\rho$ be the 35-dimensional irreducible representation of $\mathfrak{g}=\mathfrak{s l}_{3}$ of highest weight $[4,1]$. Then $\operatorname{RND}(\rho(\mathfrak{g}))$ is a sum of three irreducible modules of highest weights $[1,4],[1,1]$, and $[4,1]$. Hence, Proposition 3 cannot be applied to conclude rank-criticality. Note that by the results of Dynkin [4] the image of $\rho$ is a maximal subalgebra of $\mathfrak{s l}_{35}$, so that there is no easy argument as in the previous two examples, that shows that $\rho(\mathfrak{g})$ is not rank-critical.

Theorem 2 was first conjectured on the basis of (1). 


\section{Conclusion And Further questions}

Representations of semisimple Lie algebras yield an abundance of rank-critical matrix spaces, which suggests that the (commonly believed to be intractable) classification of such spaces may in some sense include the classification of Lie algebra representations. In particular, among the representations of $\mathfrak{s l}_{3}$ we found infinitely many where the image of $\mathfrak{s l}_{3}$ is a maximal singular space. The matrix spaces $\mathcal{A}$ constructed this way actually satisfy an a priori stronger condition than rankcriticality, namely: $\mathcal{A}=\operatorname{RND}(\mathcal{A})$. These results pose many questions for further research, of which the following seem most interesting.

(1) Directly describe, given the highest weights in a representation $\rho$ of a split semisimple Lie algebra $\mathfrak{g}$, the highest weights in the $\mathfrak{g}$-module $\operatorname{RND}(\rho(\mathfrak{g}))$. The present proofs of Theorems 1 and 2 are somewhat ad hoc, and the algorithm of Section 5 is computationally rather intensive, so that it only works for representations of dimensions at most 50 or so.

(2) Investigate the discrepancy between rank-criticality and the condition $\mathcal{A}=$ $\operatorname{RND}(\mathcal{A})$.

(3) For $m_{n}$ the minimal dimension of a maximal singular space of $n \times n$ matrices, determine $\liminf _{n \rightarrow \infty} m_{n}$ (which is larger than 2 and at most 8 , as we have seen, while - to the best of my knowledge - it was previously believed to be infinite) and $\lim _{\sup _{n \rightarrow \infty}} m_{n}$.

(4) Investigate whether the maximal singular spaces of Theorem 1 remain maximal modulo primes, and, more generally, whether rank-critical spaces constructed in Section 4 remain rank-critical modulo primes.

\section{REFERENCES}

[1] LeRoy B. Beasley. Null spaces of spaces of matrices of bounded rank. In Current trends in matrix theory, Proc. 3rd Conf., Auburn/Ala. 1986, pages 45-50. North-Holland, New York, 1987.

[2] Andries Brouwer. Automatic summation using Zeilberger-Wilf theory. Nieuw Arch. Wiskd. (5), 3(4):308-312, 2002.

[3] Jean Dieudonné. Sur une généralisation du groupe orthogonal à quatre variables. Arch. Math., Oberwolfach, 1:282-287, 1949.

[4] Eugene B. Dynkin. Maximal subgroups of the classical groups. Am. Math. Soc. Transl., II. Ser. 6:245-378, 1957.

[5] David Eisenbud and Joe Harris. Vector spaces of matrices of low rank. Adv. Math., 70(2):135155,1988

[6] P. Fillmore, C. Laurie, and H. Radjavi. On matrix spaces with zero determinant. Linear Multilinear Algebra, 18:255-266, 1985.

[7] H. Flanders. On spaces of linear transformations with bounded rank. J. Lond. Math. Soc., 37:10-16, 1962.

[8] William Fulton and Joe Harris. Representation theory. A first course. Number 129 in Graduate Texts in Mathematics. Springer-Verlag, New York, 1991.

[9] F.R. Gantmacher. The theory of matrices. Vol. 2. AMS Chelsea Publishing, New York, 1959.

[10] Boaz Gelbord and Roy Meshulam. Spaces of singular matrices and matroid parity. Eur. J. Comb., 23(4):389-397, 2002.

[11] Joe Harris. Algebraic geometry. A first course. Number 133 in Graduate Texts in Mathematics. Springer-Verlag, Berlin etc., 1992.

[12] Bo Ilic and J.M. Landsberg. On symmetric degeneracy loci, spaces of symmetric matrices of constant rank and dual varieties. Math. Ann., 314(1):159-174, 1999.

[13] Raphael Loewy. Large spaces of symmetric matrices of bounded rank are decomposable. Linear Multilinear Algebra, 48(4):355-382, 2001. 
[14] Raphael Loewy and Nizar Radwan. Spaces of symmetric matrices of bounded rank. Linear Algebra Appl., 197-198:189-215, 1994.

[15] László Lovász. Singular spaces of matrices and their application in combinatorics. Bol. Soc. Bras. Mat., Nova Sér., 20(1):87-99, 1989.

[16] Roy Meshulam. On two extremal matrix problems. Linear Algebra Appl., 114-115:261-271, 1989.

[17] Marko Petkovšek, Herbert S. Wilf, and Doron Zeilberger. A = B. A. K. Peters, Wellesley, Massachusetts, 1996.

[18] R. W. Richardson. Commuting varieties of semisimple lie algebras and algebraic groups. Compositio Math., 38(3):311-327, 1979.

Jan Draisma, Mathematisches Institut der Universität Basel, Switzerland

E-mail address: jan.draisma@unibas.ch 